\title{
Palliative Percutaneous Jejunal Stent for Patients with Short Bowel Syndrome
}

\author{
Satoru Takayama Yasuo Ochi Akira Yasuda \\ Masaki Sakamoto Hideki Takahashi Yoshimi Akamo \\ Hiromitsu Takeyama \\ Department of Gastroenterological Surgery, Nagoya City University Graduate \\ School of Medical Sciences, Nagoya, Japan
}

\section{Key Words}

Expandable metallic stent . Percutaneous endoscopic duodenostomy - Percutaneous jejunal stent $\cdot$ Ultrathin endoscopy $\cdot$ Intraoperative stent

\begin{abstract}
Gastrointestinal obstruction is a common preterminal event in patients with gastric and pancreatic cancer who often undergo palliative bypass surgery. Although endoscopic palliation with self-expandable metallic stents has emerged as a safe and effective alternative to surgery, experience with this technique remains limited. In particular, a proximal jejunal obstruction requires more technical expertise than a duodenal obstruction. Palliative treatment modalities include both surgical and nonsurgical approaches. In this report, we describe the successful placement of self-expandable metallic stents at the proximal jejunum using a combination of percutaneous endoscopic, intraoperative, and transstomal stenting. Usually endoscopy is not indicated in cases of proximal jejunal obstruction, but some cases may require palliative endoscopy instead of bypass operation.
\end{abstract}

\section{Case Report}

A 56-year-old female was admitted for nausea and vomiting. She had a history of stage IV gastric cancer and had undergone proximal gastrectomy and bilateral adnexectomy simultaneously due to metastasis of gastric cancer. In addition, she had undergone right hemicolectomy and near-total small bowel resection for bowel ischemic necrosis caused by metastatic bowel obstruction. After the operation, she had been fed through a subcutaneous intravenous hyperalimentation (IVH) port.

Past history and the presence of emesis suggested small bowel obstruction due to abdominal carcinomatosis. The day after admission, a contrast $\mathrm{x}$-ray showed a small bowel stricture at the beginning of the jejunum, for which we inserted a small transnasal small bowel tube as a palliative 
drainage pathway (ig. 1). Since the patient found the nasal and throat discomfort accompanying the transnasal tube intolerable, we performed percutaneous endoscopic duodenostomy (PED) with fixation of the duodenum to the skin but failed to reach the stomach due to postoperative gastric shortening. The skin around the duodenostomy became severely eroded due to digestive secretion leakage and the patient continued to complain of discomfort. Furthermore, serum albumin levels could not be maintained by IVH due to the massive loss of digestive enzymes. Therefore, we tried to place a jejunum expandable metallic stent. We inserted a nasal gastric camera through the PED fistula. The camera passed through the stricture and was replaced by a guidewire. The self-expandable metallic stent was inserted percutaneously over the guidewire (i.g. 2); however, the stent did not completely open the passage and the skin erosion did not heal. To correct this situation, we elected to perform the stenting by open surgery. Because of severe carcinomatosis we could not confirm a mobile jejunum, which would have been suitable for anastomosis of the bypass. Due to the stricture, we could not pass the camera through the jejunal incision. Therefore the stent was inserted through the PED fistula with a guidewire. The stent was placed at the stricture just distal to the first stent (toward the anal end). Although stent placement was successful, the operative scar became a skin fistula due to poor alimentation, and digestive secretions leaked from the scar. Next we inserted a transanal ileal tube into the duodenum through the operative fistula, and additionally inserted an endoscopic bile duct stent for obstructive jaundice. After the stenting and the insertion of a transanal ileal tube, the patient remained stable. However, she could only take liquid or paste food and digestive secretions continued to leak from the operative fistula. To correct this situation, we inserted a covered stent retrograde to the stoma (fig. 3). This minimized the leakage and the digestive secretions flowed from the stoma. Despite successful stenting, the patient died of sepsis and liver dysfunction two weeks later.

\section{Discussion}

Percutaneous endoscopic gastrostomy (PEG) has been widely used to maintain enteral nutrition, particularly in dysphagic patients diagnosed with neurological diseases such as amyotrophic lateral sclerosis, Parkinson's disease, or multiple system atrophy. PEG may also be used palliatively in patients with malignant, pyloric, or duodenal stenosis and other small bowel obstructions requiring decompression [1]. This procedure reduces abdominal discomfort, but may cause loss of digestive enzymes and ultimately hypoalimentation. If it is possible to insert a self-expandable metallic stent, this may be superior to palliative PEG decompression. Self-expandable metallic stents were originally used for esophageal stenosis and occasionally for malignant gastroduodenal obstruction [2-4]. However, due to the flexibility and limited reach of the gastroscope as well as the loop anatomy of the stomach, stent placement in the distal duodenum or proximal jejunum can be very difficult. A good option may be to use a longer stent and a colonoscope [5]. In addition, with PEG, trans-PEG stenting for the duodenum or proximal jejunum is technically easier than the normal peroral access. If the stent fails, the PEG can still be useful for palliation. Currently, transnasal esophagogastroduodenoscopy is routinely used for upper gastrointestinal examination and the availability of thin scopes is better than before. The combination of ultrathin endoscopy and PEG makes it easier to access the duodenum or proximal jejunum. However, in our case, we cut a proximal open-type stent to produce a very short tip for delivery. Furthermore, we employed double-balloon endoscopy that allowed a deep approach to the small bowel. Despite our trial, we could not insert an additional stent. We had another successful experience with intraoperative transcystic ductal common bile duct stenting for a patient of pancreatic head cancer which turned out to be inoperable after laparotomy. Therefore in this particular case, we thought intraoperative stenting with minimum laparotomy was also feasible due to the widespread cancer. Insertion of the third covered stent was effective for protecting against digestive secretion leakage, indicating that a covered stent may be useful for some fistulas. In this case, we believed that one stent would correct the situation and improve the quality of life for this patient. 
A further problem with this patient was that she had short bowel syndrome (SBS). The most common cause of intestinal failure is SBS, defined as $<200 \mathrm{~cm}$ of functional small intestine. SBS may result from congenital abnormalities or surgical resection. For the past three decades, patients with severe SBS have been managed with home parenteral nutrition [6]. This patient had an IVH port and was receiving home parenteral nutrition, but there were some difficulties related to the SBS, including slow healing of the PED fistula and the small incision made for the stenting procedure. In addition, long-term continuous hyperalimentation resulted in unstable blood glucose levels, followed by hypoglycemic syncope, infectious sepsis, and death. This was a very rare case of proximal jejunal stenting for a patient with SBS.

Fig. 1. Percutaneous contrast imaging of small bowel tube demonstrating a proximal jejunal stricture.

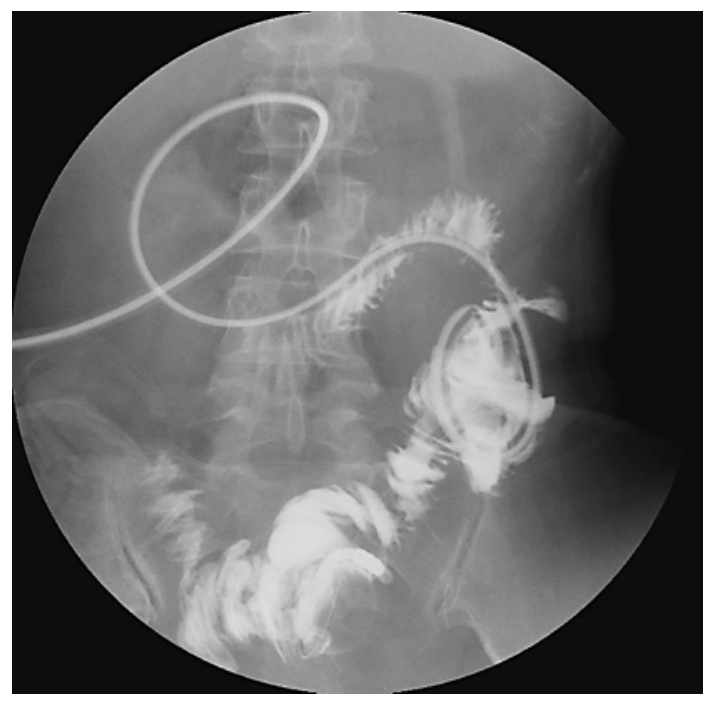


Fig. 2. Percutaneous ultrathin endoscopic image showing a covered stent placed at the proximal jejunum stricture.

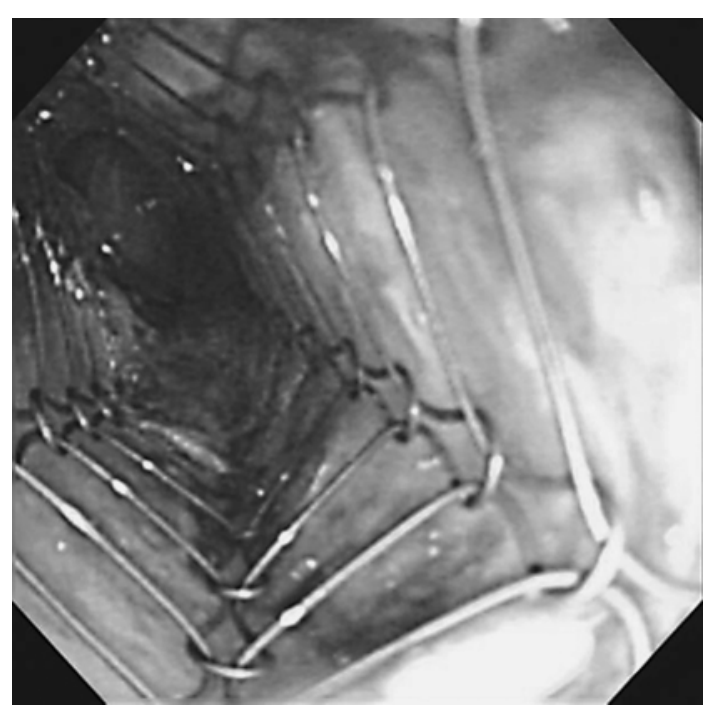

Fig. 3. Placement of a covered stent at the fistula, which prevented the leakage of digestive secretions. A cholangio-ductal stent can also be seen.

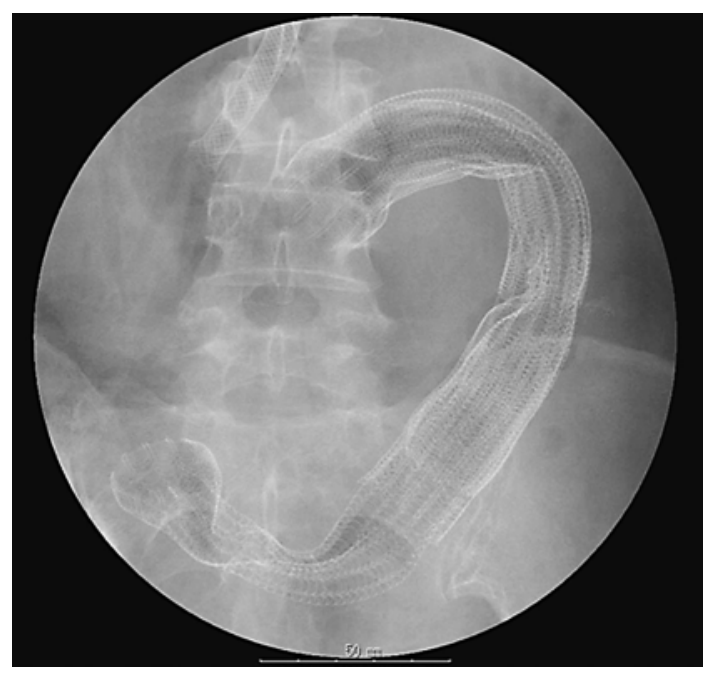




\section{References}

1 Holm AN, Baron TH: Palliative use of percutaneous endoscopic gastrostomy and percutaneous endoscopic cecostomy tubes. Gastrointest Endosc Clin North Am 2007;17:795-803.

2 Bessoud B, de Baere T, Denys A, Kuoch V, Ducreux M, Precetti S, Roche A, Menu Y: Malignant gastroduodenal obstruction: palliation with self-expanding metallic stents. J Vasc Interv Radiol 2005;16:247-253.

3 Brooksbank MA, Game PA, Ashby MA: Palliative venting gastrostomy in malignant intestinal obstruction. Palliat Med 2002;16:520-526.

4 Holt AP, Patel M, Ahmed MM: Palliation of patients with malignant gastroduodenal obstruction with self-expanding metallic stents: the treatment of choice? Gastrointest Endosc 2004;60:1010-1017.

5 Jeurnink SM, Repici A, Luigiano C, Pagano N, Kuipers EJ, Siersema PD: Use of a colonoscope for distal duodenal stent placement in patients with malignant obstruction. Surg Endosc 2009;23:562-567.

-6 Matarese LE, O'Keefe SJ, Kandil HM, Bond G, Costa G, Abu-Elmagd K: Short bowel syndrome: clinical guidelines for nutrition management. Nutr Clin Pract 2005;20:493-502. 